\title{
Assessment of Formula-Based Structural Annotation of Humic Substances by Mild Chemical Derivatization and Mass Spectrometry
}

\section{Mikhnevich T. A. ${ }^{1,2}$, Zaitsev K. V. ${ }^{2}$, Perminova I. V. ${ }^{2}$ and Zherebker A. ${ }^{1}$}

${ }^{1}$ Skolkovo Institute of Science and Technology, Skolkovo, Moscow region, Russia, tanya.4erkasova8q@gmail.com

'Lomonosov Moscow State University, Department of Chemistry, Moscow, Russia doi: 10.36291/HIT.2021.mikhnevich.002

Natural organic matter (NOM) plays an important role in the environment and its chemical properties and molecular composition reflect balance between mineralization and sequestration of organic carbon. Ultrahigh resolution mass spectrometry (e.g., FTICR MS) provides essential molecular information about NOM. However, NOM molecular heterogeneity prevents application of tandem MS experiments and direct structural information is ultimately missing leaving opportunities to only ambiguous formula-based annotation. The main aim of this work was to develop a chemical workflow to reliably examine the accuracy of several FTICR MS-derived structural indices with the focus on aromaticity and O-functional groups, which greatly impact compound properties.

Four NOM samples of different origin (coal, oxidized lignin, river, and permafrost thaw) were brominated by NBS in acetonitrile for $24 \mathrm{hrs}$ at RT. Carboxylic groups in all samples were determined by selective deuteromethylation using $\mathrm{CD}_{3} \mathrm{OD} / \mathrm{SOCl}_{2}$ reaction and by HATU amidation with ${ }^{15} \mathrm{~N}$ labeled glycine. Carbonyl groups were reduced by NaBD4. All parent and labeled mixtures were analyzed by ESI FTCR MS. Custom python scripts were developed to treat spectra and enumerate specific structural moieties in individual components. Obtained data was used to assess reliability of exact aromaticity indices $(\mathrm{Al})^{1}$ and aromaticity equivalents $\left(\mathrm{X}_{\mathrm{c}}\right)^{2}$.

Lignin- and coal-derived samples turned out to be the most sensitive to bromination which corroborated with the model phenolic structures. On contrary, permafrost thaw, which is enriched with labile species, was mostly resistant to bromination - $22 \%$ of molecular ions were brominated. Moreover, unlike oxidized riverine sample, coal NOM included polybrominated species, which implies that reaction efficiency depends on reactivity (i.e. substituents) of aromatic fragments. Samples were characterized by drastically different bromine distributions on van Krevelen diagrams, which correlated with the distribution of non-carboxylic oxygen atoms. Further, we compared $\mathrm{Al}$ and $\mathrm{X}_{\mathrm{c}}$ aromaticity indices in terms of the proportion of correctly assigned aromatics. The data on brominated molecules were in good agreement with the Al values; however, apparently Al tends to overestimate the number of non-aromatics in the sample since it describe averaged aromaticity rather than the factual presence of aromatic ring. On the other hand, $X_{c}$ perfectly recognized non-aromatics. In general, a higher proportion of correctly attributed aromatics was observed for the aromaticity equivalent $X_{c}$ (up to $68 \%$ ), which tends to find aromatic moieties in non-aromatic molecules assigned by Al. Still, we observed a number of aromatic- and condensed aromatic-assigned compounds, which were resistant to bromination or included lesser Br-atoms than the evaluated number of aromatic rings. Reaction with $\mathrm{NaBD}_{4}$ and enumeration of labeling series revealed the presence of carbonyl groups in these species, which in case of multiple reducing could be reliably assigned to quinone - condensed non-aromatic compounds. The approach may be of great importance in biogeochemical and medicinal studies of NOM.

Acknowledgements. This work was supported by the Russian Science Foundation gran № 21-47-04405.

References

1. Zherebker, A., Lechtenfeld, O. J., Sarycheva, A., Kostyukevich, Y., Kharybin, O., Fedoros, E. I. and Nikolaev, E. N. Anal. Chem., 2020, 92 (13), 9032-9038;

2. Yassine, M.M., Harir, M., Dabek-Zlotorzynska, E. and Schmitt-Kopplin, P. Rapid Commun. Mass Spectrom., 2014, 28, 2445-2454. 